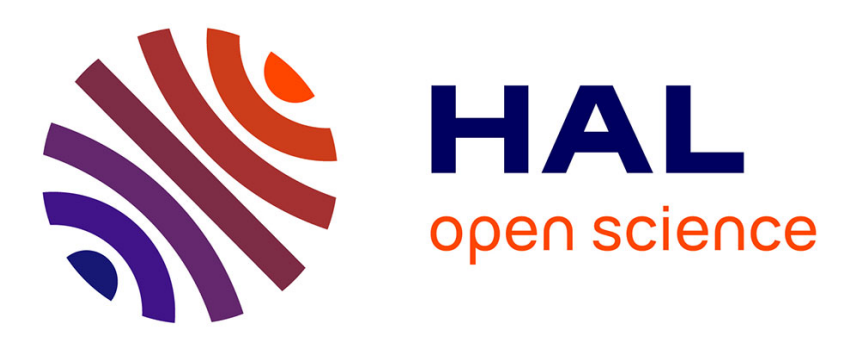

\title{
PARATUCK-3 Semi-Blind Receivers for Three-Hop Cooperative MIMO Relay Systems
}

\author{
Pedro Marinho R. de Oliveira, C. Alexandre Rolim Fernandes
}

\section{To cite this version:}

Pedro Marinho R. de Oliveira, C. Alexandre Rolim Fernandes. PARATUCK-3 Semi-Blind Receivers for Three-Hop Cooperative MIMO Relay Systems. XXXV Simpósio Brasileiro de Telecomunicações e Processamento de Sinais, Sep 2017, São Pedro, Brazil. 10.14209/sbrt.2017.245 hal-01954369

\section{HAL Id: hal-01954369 \\ https://hal.science/hal-01954369}

Submitted on 13 Dec 2018

HAL is a multi-disciplinary open access archive for the deposit and dissemination of scientific research documents, whether they are published or not. The documents may come from teaching and research institutions in France or abroad, or from public or private research centers.
L'archive ouverte pluridisciplinaire HAL, est destinée au dépôt et à la diffusion de documents scientifiques de niveau recherche, publiés ou non, émanant des établissements d'enseignement et de recherche français ou étrangers, des laboratoires publics ou privés. 


\title{
PARATUCK-3 Semi-Blind Receivers for Three-Hop Cooperative MIMO Relay Systems
}

\author{
Pedro M. R. de Oliveira and C. Alexandre Rolim Fernandes
}

\begin{abstract}
In this paper, it is considered a three-hop oneway AF cooperative communication system, using a Khatri-Rao Space-Time (KRST) coding at the source. It is shown that the third-order tensor of signals received at the destination node satisfies a PARATUCK-3 tensor model. This tensorial modeling enables a joint semi-blind estimation of the transmitted symbols, the relay-destination channel and a global channel that contains the gains of the other channels. Two non-iterative estimation algorithms based on the Kronecker product are then proposed. The performance of these receivers is evaluated by means of computational simulation results.
\end{abstract}

Keywords - Cooperative Systems, MIMO, PARATUCK-3, Kronecker Product, Semi-Blind Receivers.

\section{INTRODUCTION}

Aiming to provide a considerable increase in the received power and a better signal quality, the concept of cooperative communications relay systems was developed, in which at least one relay node is used to assist the communication between the source node and the destination node [1]. A cooperative communication can be done according to several protocols, that are classified in fixed (Amplify-and-Forward (AF), Decode-and-Forward (DF), Compress-and-Forward (CF), and others) and adaptive (selective decode-and-forward, incremental relaying, and others). In particular, multi-hop systems have the advantage of needing less transmission power than twohop networks, as the distance between the source and the destination is divided in several smaller links.

Moreover, Multiple-Input Multiple-Output (MIMO) systems provided a great advance in the wireless communication field, due to its considerable increase in both the coverage area and the capacity. This technology quickly developed and is an important research field nowadays, being present in several standards (WIMAX-IEEE 802.16, WLAN-IEEE 802.11N, and many others) [2].

On the other hand, due to its advantages in exploring the multidimensional nature of a problem, tensor decompositions are applied in several areas, including digital signal processing [3], [4], [5] and telecommunications [6], [7], [8]. Also, tensor analysis has shown to be an efficient approach for channel and/or symbol estimation in cooperative MIMO systems [9], [10], [11], [12], [13].

In [9], it was proposed three semi-blind receivers in a twohop MIMO AF relaying system using the Khatri-Rao SpaceTime (KRST) coding [14]. These receivers combine two tensor

Pedro M. R. de Oliveira is with the Department of Teleinformatics Engineering, Federal University of Ceará, Fortaleza, Brazil. (e-mail: pedromarinh8@gmail.com). C. Alexandre Rolim Fernandes is with the Department of Computer Engineering, Federal University of Ceará, Sobral, Brazil. (e-mail: alexandre_ufc@yahoo.com.br). This work was partially supported by CNPq and CAPES. models (PARAllel FACtor - PARAFAC and PARATUCK2 ), that enables the joint estimation of the symbols and the channels of the source-relay and relay-destination links. Also in a two-hop MIMO AF relaying system, in the work [10] a Nested-PARAFAC tensor model is formulated and two iterative semi-blind receivers are proposed, jointly estimating the symbol and the channels of the communication links. In [12] a Tensor Space-Time (TST) coding is used in MIMO wireless communication systems, while in [11] this TST coding is used with a Nested Tucker decomposition in a MIMO relaying system. A blind receiver based on a generalized PARATUCK-2 decomposition is proposed in [13] for Space-Time Frequency (STF) MIMO systems.

Due to its simplicity, in this work it is considered an AF relaying system. More specifically, it is considered a three-hop scenario, with a multiple-antenna source node, two multipleantenna AF relay nodes and a multiple-antenna destination node. This scenario is an one-way half-duplex relaying system, where the source node transmits to the destination during three consecutive transmission phases.

This work proposes two semi-blind receivers based on the Kronecker product, that jointly estimate the symbol and the channels in a three-hop AF MIMO relay-assisted system. We consider a transmission scheme using a simplified KRST coding [14] at the source node, combined with an AF coding scheme at the relay nodes. We show that the third-order tensor of signals received by the destination node satisfies a PARATUCK-3 decomposition [15]. This tensorial modeling enables a semi-blind estimation of symbols and channels with the use of a few pilot symbols. We propose two non-iterative receivers algorithms that are based on the Kronecker product. In the first one, Singular-Value Decomposition (SVD) is used to jointly estimate the symbol, the channel of the last hop link and a global channel that contains information of the other links. The second one, is based on an estimation algorithm proposed in [16], in which a rearrangement of the Kronecker product matrix is done in order to achieve a rank-1 matrix. The symbols and the matrix of the last hop are estimated using the SVD of this rank-1 matrix, and a the so-called global channel is estimated as the first algorithm. These algorithms have the advantage of being non-iterative, providing a good performance. Also, the multi-hop cooperative systems provides an additional advantage due to its transmission power gain, as previously described.

The rest of this paper is organized as follows. In Section II, the notations used are defined and two important matrix properties are recalled. In Section III, the system model for the present scenario is described. In Section IV, two noniterative receivers algorithms are proposed. In Section $\mathrm{V}$ the 


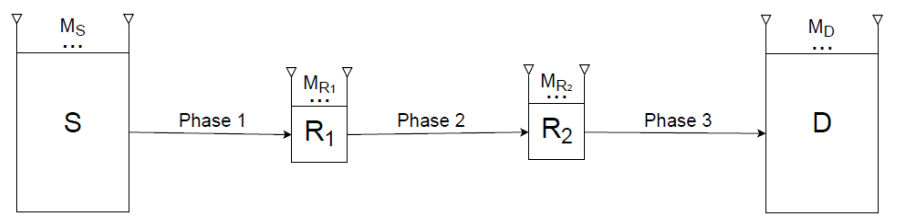

Fig. 1: MIMO cooperative system model.

computational simulation results are presented and finally, the Section VI presents the conclusion of this work, as well as the prospects for future works.

\section{NOTATIONS}

Scalars, vectors, matrices, and tensors are represented, respectively, by lower-case $(a, b, c, \ldots)$, boldface lower-case (a, $\mathbf{b}, \mathbf{c}, \ldots)$, boldface capital $(\mathbf{A}, \mathbf{B}, \mathbf{C}, \ldots)$, and calligraphic $(\mathcal{A}$, $\mathcal{B}, \mathcal{C}, \ldots)$ letters.

The transpose, the Hermitian transpose, the Moore-Penrose pseudo-inverse, the conjugate, the $m^{\text {th }}$ row and the $n^{\text {th }}$ column of $\mathbf{A} \in \mathbb{C}^{M \times N}$, are respectively represented by $\mathbf{A}^{T}$, $\mathbf{A}^{H}, \mathbf{A}^{\dagger}, \mathbf{A}^{*}, \mathbf{A}_{m}$. and $\mathbf{A}_{. n}$. The $\|\cdot\|_{F}$ represents the Frobenius norm, $\otimes$ represents the Kronecker product and $\diamond$ represents the Khatri-Rao (column-wise Kronecker) product. The operator $\operatorname{diag}(\mathbf{a})$ generates a diagonal matrix with the vector argument a forming its diagonal, $D_{m}(\mathbf{A})$ represents the diagonal matrix with the $m^{\text {th }}$ row of $\mathbf{A}$ forming its diagonal, the operator $\operatorname{vec}(\cdot)$ vectorizes its matrix argument by stacking its columns, while the operator unvec $(\cdot)$ unvectorizes its vector argument to the original matrix. The operator $S V D(\mathbf{A})$ computes the singular-value decomposition of its matrix argument $\mathbf{A}$

In this work, it were used the two following properties

$$
\begin{gathered}
\operatorname{vec}\left(\mathbf{A B C} \mathbf{C}^{T}\right)=(\mathbf{C} \otimes \mathbf{A}) \operatorname{vec}(\mathbf{B}) . \\
D_{p}(\mathbf{A}) \otimes D_{p}(\mathbf{B})=D_{p}\left(\left(\mathbf{A}^{T} \diamond \mathbf{B}^{T}\right)^{T}\right) .
\end{gathered}
$$

Given a third-order tensor $\mathcal{A} \in \mathbb{C}^{M \times N \times P}$, with scalars $a_{m, n, p}$, its horizontal, lateral and frontal slices are respectively represented by $\mathbf{A}_{m . .} \in \mathbb{C}^{N \times P}, \mathbf{A}_{. n .} \in \mathbb{C}^{P \times M}$ and $\mathbf{A}_{. . p} \in$ $\mathbb{C}^{M \times N}$. Given a matrix $\mathbf{A} \in \mathbb{C}^{I \times J}, a_{i j}$ represents its $i j^{t h}$ element.

\section{SySTEM MODEL}

For the present work, it is considered a three-hop one-way cooperative MIMO AF relay system, with a source $(S)$ node, a destination $(D)$ node and two relays $\left(R_{1}\right.$ and $\left.R_{2}\right)$ nodes, as illustrated in Figure 1, where $M_{X}$ denotes the number of antennas at node $X$ (e.g, $M_{S}$ denotes the number of antennas at node $S$ ). All the channels are assumed to be invariant during the total transmission time and to undergo frequency flat fading. The transmitted symbols are QAM-modulated.

Consider that $\mathbf{H}^{\left(S R_{1}\right)} \in \mathbb{C}^{M_{R_{1}} \times M_{S}}, \mathbf{H}^{\left(R_{1} R_{2}\right)} \in \mathbb{C}^{M_{R_{2}} \times M_{R_{1}}}$ and $\mathbf{H}^{\left(R_{2} D\right)} \in \mathbb{C}^{M_{D} \times M_{R_{2}}}$ are MIMO channel matrices of the source-relay ${ }^{1}$, relay $^{1}$-relay ${ }^{2}$ and the relay ${ }^{2}$-destination links, respectively. $\mathbf{S} \in \mathbb{C}^{N \times M_{S}}$ is the matrix with the information symbols multiplexed to the $M_{S}$ antennas during $N$ consecutive symbol periods. A simplified KRST coding [14] is used at the source to introduce time redundancy:

$$
\mathbf{X}_{. . p}=D_{p}\left(\mathbf{G}_{0}\right) \mathbf{S}^{T} \in \mathbb{C}^{M_{S} \times N}
$$

where $p=1, \ldots, P, \mathbf{X}_{. . p}$ is the transmitted signal, $\mathbf{G}_{0} \in$ $\mathbb{C}^{P \times M_{S}}$ is the coding matrix of the source node, and $P$ is the number of transmission blocks. The signal received by the relay $R_{1}$ during the $p^{t h}$ transmission block is given by

$$
\tilde{\mathbf{Y}}_{. . p}^{\left(S R_{1}\right)}=\mathbf{H}^{\left(S R_{1}\right)} \mathbf{X}_{. . p}+\mathbf{V}_{. . p}^{\left(S R_{1}\right)} \in \mathbb{C}^{M_{R_{1}} \times N},
$$

where $\mathbf{V}_{. . p}^{\left(S R_{1}\right)} \in \mathbb{C}^{M_{R_{1}} \times N}$ is the Additive White Gaussian Noise (AWGN) matrix during the $p^{t h}$ transmission block in the $S R_{1}$ link. Considering $\mathbf{G}_{1} \in \mathbb{C}^{P \times M_{R_{1}}}$ the coding matrix of the relay ${ }^{1}$ node, the amplified signal $D_{p}\left(\mathbf{G}_{1}\right) \tilde{\mathbf{Y}}_{\text {..p }}^{\left(S R_{1}\right)} \in$ $\mathbb{C}^{M_{R_{1}} \times N}$ is transmitted by the relay $R_{1}$ to the relay $R_{2}$.

The signal received by the relay $R_{2}$ during the $p^{\text {th }}$ transmission block can then be written as

$\tilde{\mathbf{Y}}_{. . p}^{\left(S R_{1} R_{2}\right)}=\mathbf{H}^{\left(R_{1} R_{2}\right)} D_{p}\left(\mathbf{G}_{1}\right) \tilde{\mathbf{Y}}_{. . p}^{\left(S R_{1}\right)}+\mathbf{V}_{. . p}^{\left(R_{1} R_{2}\right)} \in \mathbb{C}^{M_{R_{2}} \times N}$.

where $\mathbf{V}_{. . p}^{\left(R_{1} R_{2}\right)} \in \mathbb{C}^{M_{R_{2}} \times N}$ is the noise matrix during the $p^{t h}$ transmission block in the $R_{1} R_{2}$ link. Then, considering $\mathbf{G}_{2} \in \mathbb{C}^{P \times M_{R_{2}}}$ the coding matrix of the relay ${ }^{2}$ node, the amplified signal $D_{p}\left(\mathbf{G}_{2}\right) \tilde{\mathbf{Y}}_{. . p}^{\left(S R_{1} R_{2}\right)}$ is transmitted by the relay $R_{2}$ to the destination.

Finally, we have that the signal received by the destination during the $p^{\text {th }}$ transmission block is

$\tilde{\mathbf{Y}}_{. . p}^{\left(S R_{1} R_{2} D\right)}=\mathbf{H}^{\left(R_{2} D\right)} D_{p}\left(\mathbf{G}_{2}\right) \tilde{\mathbf{Y}}_{. . p}^{\left(S R_{1} R_{2}\right)}+\mathbf{V}_{. . p}^{\left(R_{2} D\right)} \in \mathbb{C}^{M_{D} \times N}$

where $\mathbf{V}_{. . p}^{\left(R_{2} D\right)} \in \mathbb{C}^{M_{D} \times N}$ is the noise matrix during the $p^{t h}$ transmission block in the $R_{2} D$ link.

Substituting (3), (4) and (5) into (6), we get:

$$
\begin{gathered}
\tilde{\mathbf{Y}}_{. . p}^{\left(S R_{1} R_{2} D\right)}=\mathbf{H}^{\left(R_{2} D\right)} D_{p}\left(\mathbf{G}_{2}\right) \mathbf{H}^{\left(R_{1} R_{2}\right)} D_{p}\left(\mathbf{G}_{1}\right) \mathbf{H}^{\left(S R_{1}\right)} \\
D_{p}\left(\mathbf{G}_{0}\right) \mathbf{S}^{T}+\mathbf{H}^{\left(R_{2} D\right)} D_{p}\left(\mathbf{G}_{2}\right) \mathbf{H}^{\left(R_{1} R_{2}\right)} D_{p}\left(\mathbf{G}_{1}\right) \mathbf{V}_{. p}^{\left(S R_{1}\right)}+ \\
\mathbf{H}^{\left(R_{2} D\right)} D_{p}\left(\mathbf{G}_{2}\right) \mathbf{V}_{. . p}^{\left(R_{1} R_{2}\right)}+\mathbf{V}_{. . p}^{\left(R_{2} D\right)} \in \mathbb{C}^{M_{D} \times N} .
\end{gathered}
$$

In other words

$$
\tilde{\mathbf{Y}}_{. . p}^{\left(S R_{1} R_{2} D\right)}=\mathbf{Y}_{. . p}^{\left(S R_{1} R_{2} D\right)}+\mathbf{V}_{. . p}^{\left(S R_{1} R_{2} D\right)} \in \mathbb{C}^{M_{D} \times N},
$$

where $\mathbf{Y}_{. . p}^{\left(S R_{1} R_{2} D\right)}$ is the noiseless signal given by

$$
\begin{array}{r}
\mathbf{Y}_{. . p}^{\left(S R_{1} R_{2} D\right)}=\mathbf{H}^{\left(R_{2} D\right)} D_{p}\left(\mathbf{G}_{2}\right) \mathbf{H}^{\left(R_{1} R_{2}\right)} D_{p}\left(\mathbf{G}_{1}\right) \mathbf{H}^{\left(S R_{1}\right)} \\
D_{p}\left(\mathbf{G}_{0}\right) \mathbf{S}^{T} \in \mathbb{C}^{M_{D} \times N}
\end{array}
$$

and $\mathbf{V}_{. . p}^{\left(S R_{1} R_{2} D\right)}$ is the global noise given by

$$
\begin{gathered}
\mathbf{V}_{. . p}^{\left(S R_{1} R_{2} D\right)}=\mathbf{H}^{\left(R_{2} D\right)} D_{p}\left(\mathbf{G}_{2}\right) \mathbf{H}^{\left(R_{1} R_{2}\right)} D_{p}\left(\mathbf{G}_{1}\right) \mathbf{V}_{. . p}^{\left(S R_{1}\right)}+ \\
\mathbf{H}^{\left(R_{2} D\right)} D_{p}\left(\mathbf{G}_{2}\right) \mathbf{V}_{. . p}^{\left(R_{1} R_{2}\right)}+\mathbf{V}_{. . p}^{\left(R_{2} D\right)} \in \mathbb{C}^{M_{D} \times N} .
\end{gathered}
$$

In order to have a better presentation of the proposed algorithms, from now on, we will ignore the noise component in (8). Equation (9) corresponds to a PARATUCK-3 decomposition [15] that can be rewritten in scalar form as

$$
\begin{aligned}
y_{m_{D}, n, p}^{\left(S R_{1} R_{2} D\right)}= & \sum_{m_{R_{2}}=1}^{M_{R_{2}}} \sum_{m_{R_{1}}=1}^{M_{R_{1}}} \sum_{m_{S}=1}^{M_{S}} h_{m_{D}, m_{R_{2}}}^{\left(R_{2} D\right)} g_{p, m_{R_{2}}}^{(2)} \\
& h_{m_{R_{2}}, m_{R_{1}}}^{\left(R_{1} R_{2}\right)} g_{p, m_{R_{1}}}^{(1)} h_{m_{R_{1}}, m_{S}}^{\left(S R_{1}\right)} g_{p, m_{S}}^{(0)} s_{n, m_{S}} .
\end{aligned}
$$




\section{Symbol AND Channels Estimation}

Before presenting the proposed algorithms, we will develop some mathematical expressions from the received signal matrix (9). Applying Property (1) in (9) twice, we have:

$$
\begin{array}{r}
\mathbf{y}_{p}^{\left(S R_{1} R_{2} D\right)}=\left(\mathbf{S} \otimes \mathbf{H}^{\left(R_{2} D\right)}\right) \operatorname{vec}\left(D_{p}\left(\mathbf{G}_{2}\right) \mathbf{H}^{\left(R_{1} R_{2}\right)} D_{p}\left(\mathbf{G}_{1}\right)\right. \\
\left.\mathbf{H}^{\left(S R_{1}\right)} D_{p}\left(\mathbf{G}_{0}\right)\right),
\end{array}
$$

$$
\begin{aligned}
\mathbf{y}_{p}^{\left(S R_{1} R_{2} D\right)} & =\left(\mathbf{S} \otimes \mathbf{H}^{\left(R_{2} D\right)}\right)\left(D_{p}\left(\mathbf{G}_{0}\right) \otimes D_{p}\left(\mathbf{G}_{2}\right)\right) \\
& \operatorname{vec}\left(\mathbf{H}^{\left(R_{1} R_{2}\right)} D_{p}\left(\mathbf{G}_{1}\right) \mathbf{H}^{\left(S R_{1}\right)}\right) \in \mathbb{C}^{M_{D} N \times 1},
\end{aligned}
$$

where $\mathbf{y}_{p}^{\left(S R_{1} R_{2} D\right)}=\operatorname{vec}\left(\mathbf{Y}_{. . p}^{\left(S R_{1} R_{2} D\right)}\right)$.

Now applying Property (2) in (13) we have:

$$
\begin{aligned}
\mathbf{y}_{p}^{\left(S R_{1} R_{2} D\right)} & =\left(\mathbf{S} \otimes \mathbf{H}^{\left(R_{2} D\right)}\right) D_{p}\left(\left(\mathbf{G}_{0}^{T} \diamond \mathbf{G}_{2}^{T}\right)^{T}\right) \\
& \operatorname{vec}\left(\mathbf{H}^{\left(R_{1} R_{2}\right)} D_{p}\left(\mathbf{G}_{1}\right) \mathbf{H}^{\left(S R_{1}\right)}\right) \in \mathbb{C}^{M_{D} N \times 1},
\end{aligned}
$$

Let us define $\mathbf{G}_{02}=\left(\mathbf{G}_{0}^{T} \diamond \mathbf{G}_{2}^{T}\right) \in \mathbb{C}^{M_{R_{2}} M_{S} \times P}$. We may then write

$$
\begin{array}{r}
\mathbf{y}_{p}^{\left(S R_{1} R_{2} D\right)}=(\mathbf{S} \otimes \\
\left.\mathbf{H}^{\left(R_{2} D\right)}\right) \operatorname{diag}\left[\operatorname { v e c } \left(\mathbf{H}^{\left(R_{1} R_{2}\right)} D_{p}\left(\mathbf{G}_{1}\right)\right.\right. \\
\left.\left.\mathbf{H}^{\left(S R_{1}\right)}\right)\right]\left(\left(\mathbf{G}_{02}^{T}\right)^{T}\right)_{p .} \in \mathbb{C}^{M_{D} N \times 1},
\end{array}
$$

Applying Property (1) in (15) we get:

$$
\begin{array}{r}
\mathbf{y}_{p}^{\left(S R_{1} R_{2} D\right)}=\left(\mathbf{S} \otimes \mathbf{H}^{\left(R_{2} D\right)}\right) \operatorname{diag}\left[\left(\mathbf{H}^{\left(S R_{1}\right)^{T}} \otimes \mathbf{H}^{\left(R_{1} R_{2}\right)}\right)\right. \\
\left.\operatorname{vec}\left(D_{p}\left(\mathbf{G}_{1}\right)\right)\right]\left(\mathbf{G}_{02}\right)_{. p} \in \mathbb{C}^{M_{D} N \times 1},
\end{array}
$$

which leads to

$$
\begin{array}{r}
\mathbf{y}_{p}^{\left(S R_{1} R_{2} D\right)}=\left(\mathbf{S} \otimes \mathbf{H}^{\left(R_{2} D\right)}\right) \operatorname{diag}\left[\left(\mathbf{H}^{\left(S R_{1}\right)^{T}} \diamond \mathbf{H}^{\left(R_{1} R_{2}\right)}\right)\right. \\
\left.\left(\mathbf{G}_{1}\right)_{p .}\right]\left(\mathbf{G}_{02}\right)_{. p} \in \mathbb{C}^{M_{D} N \times 1} .
\end{array}
$$

Assuming that the rows of $\mathbf{G}_{1}$ are equal, i.e, $\left(\mathbf{G}_{1}\right)_{p \text {. }}=\mathbf{g}_{1}$, for $p=1, \ldots, P$, we have:

$$
\begin{array}{r}
\mathbf{y}_{p}^{\left(S R_{1} R_{2} D\right)}=\left(\mathbf{S} \otimes \mathbf{H}^{\left(R_{2} D\right)}\right) \operatorname{diag}\left[\left(\mathbf{H}^{\left(S R_{1}\right)^{T}} \diamond \mathbf{H}^{\left(R_{1} R_{2}\right)}\right) \mathbf{g}_{1}\right] \\
\left(\mathbf{G}_{02}\right)_{. p} \in \mathbb{C}^{M_{D} N \times 1}, \quad
\end{array}
$$

By stacking the vectors $\mathbf{y}_{p}^{\left(S R_{1} R_{2} D\right)}$ for $p=1, \ldots, P$, we get the mode-1 unfolded matrix of the third-order tensor $\mathcal{Y}^{\left(S R_{1} R_{2} D\right)} \in \mathbb{C}^{M_{D} \times N \times P}$ :

$$
\mathbf{Y}_{1}^{\left(S R_{1} R_{2} D\right)}=\left[\mathbf{y}_{1}^{\left(S R_{1} R_{2} D\right)} \ldots \mathbf{y}_{P}^{\left(S R_{1} R_{2} D\right)}\right]
$$

then

$$
\begin{array}{r}
\mathbf{Y}_{1}^{\left(S R_{1} R_{2} D\right)}=\left(\mathbf{S} \otimes \mathbf{H}^{\left(R_{2} D\right)}\right) \operatorname{diag}\left[\left(\mathbf{H}^{\left.\left.\left(S R_{1}\right)^{T} \diamond \mathbf{H}^{\left(R_{1} R_{2}\right)}\right) \mathbf{g}_{1}\right]}\right.\right. \\
\mathbf{G}_{02} \in \mathbb{C}^{M_{D} N \times P}, \quad(20)
\end{array}
$$

Let us assume that $\mathbf{G}_{02}$ has a right inverse, i.e. $\mathbf{G}_{02} \mathbf{G}_{02}^{\dagger}=\mathbf{I}$. This means that the rank of $\mathbf{G}_{02}$ is equal to $M_{R_{2}} M_{S}$, which implies $P \geq M_{R_{2}} M_{S}$, so

$$
\mathbf{Y}_{2}^{\left(S R_{1} R_{2} D\right)} \mathbf{G}_{02}^{\dagger}=\left(\mathbf{S} \otimes \mathbf{H}^{\left(R_{2} D\right)}\right) \operatorname{diag}\left[\mathbf{h}^{(G)}\right] \in \mathbb{C}^{M_{D} N \times P},
$$

where

$$
\mathbf{h}^{(G)}=\left(\mathbf{H}^{\left(S R_{1}\right)^{T}} \diamond \mathbf{H}^{\left(R_{1} R_{2}\right)}\right) \mathbf{g}_{1} \in \mathbb{C}^{M_{R_{2}} M_{S} \times 1} .
$$

is the vectorized form of the so-called global channel $\mathbf{H}^{(G)}$, as it depends on both channel matrices $\mathbf{H}^{\left(S R_{1}\right)}$ and $\mathbf{H}^{\left(R_{1} R_{2}\right)}$.

In the next two subsections, (21) will be used to jointly estimate the symbol matrix $\mathbf{S}$ and the channels $\mathbf{H}^{\left(R_{2} D\right)}$ and $\mathbf{H}^{(G)}$ (i.e unvec $\left(\mathbf{h}^{(G)}\right)$ ) by means of two non-iterative algorithms based on the Kronecker product. We propose two different semi-blind receivers to estimate these matrices. In this work, we assume that the coding matrices $\mathbf{G}_{0}, \mathbf{G}_{1}$ and $\mathbf{G}_{2}$ are known at the destination node.

\section{A. Least-Squares Kronecker Factorization (LS-KF) Algorithm}

Let us define $\mathbf{W}$ as

$$
\mathbf{W}=\left(\mathbf{S} \otimes \mathbf{H}^{\left(R_{2} D\right)}\right) \operatorname{diag}\left(\mathbf{h}^{(G)}\right) \in \mathbb{C}^{M_{D} N \times M_{R_{2}} M_{S}} .
$$

The first step of the LS-KF algorithm consists in estimating W from (21) by means of the Least Squares (LS) method. Then, we estimate $\mathbf{S}$ and $\mathbf{H}^{\left(R_{2} D\right)}$ using several SVDs of the columns of $\mathbf{W}^{\text {est }}$ (the LS estimate of $\mathbf{W}$ ). This procedure, denoted Kronecker Factorization $(\mathrm{KF})$, is based on the fact that $\operatorname{unvec}\left(\mathbf{W}_{. j}\right)=\mathbf{h}_{{ }_{.}{ }_{r}}^{\left(R_{2} D\right)} \mathbf{S}_{. i}^{T} h_{j}^{(G)}$, with $j=(i-1) M_{R_{2}}+$ $m_{r}$, is a rank-1 matrix, which means that $\mathbf{S}_{. i}$ and $\mathbf{h}_{m_{r}}^{\left(R_{2} D\right)}$, with $i=1, \ldots, M_{S}, m_{r}=1, \ldots, M_{R_{2}}$, are optimally estimated as the first right- and left-singular vectors of unvec $\left(\mathbf{W}_{. j}^{e s t}\right)$, respectively.

At the end, we will have $M_{S}$ estimations of $\mathbf{S}$ and $M_{R_{2}}$ estimations of $\mathbf{H}^{\left(R_{2} D\right)}$ with scalar ambiguities in each column of these matrices. We take the first of these estimations. We cannot estimate these matrices without ambiguities, they are inherent to the model. We can eliminate the ambiguities of $\mathbf{S}$ assuming that we know its first row, this is possible by using one pilot symbol by transmission stream. In the $\mathbf{H}^{\left(R_{2} D\right)}$ case, we cannot eliminate its ambiguity, however, we assume that we know its first row just to plot the simulation results. The global channel matrix $\mathbf{H}^{(G)}$ is estimated with ambiguities using the first row of $\mathbf{W}^{\text {est }}$. Such ambiguities cannot be eliminated, once we do not have knowledge of the channels contained in the global channel. The LS-KF algorithm is summarized in Algorithm 1.

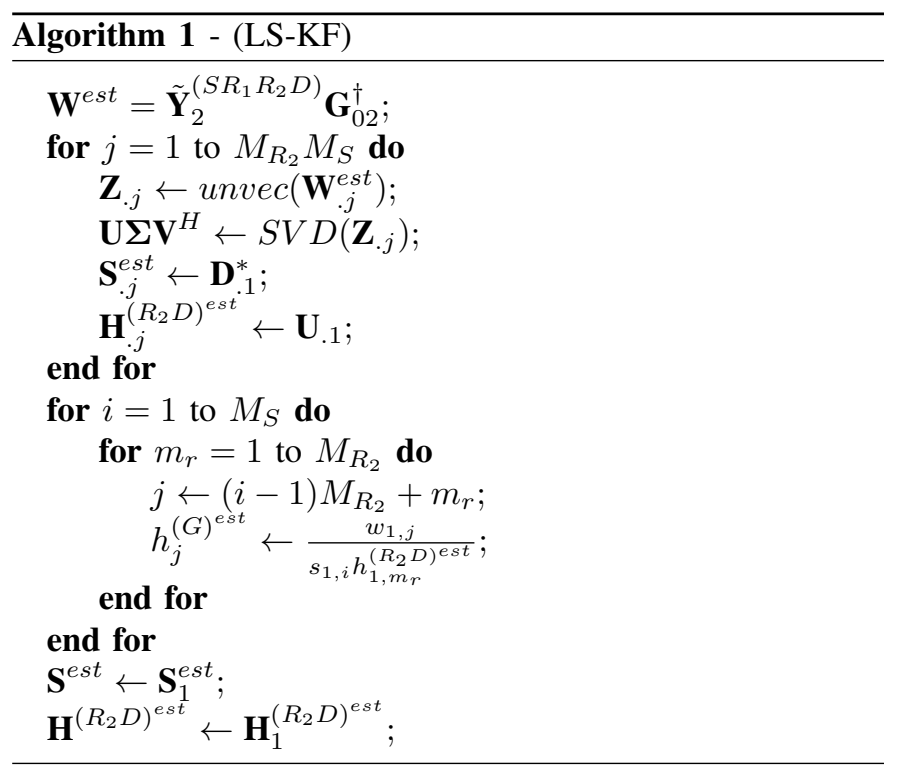




\section{B. Least-Squares Kronecker Rearrangement-Based (LS-KR) Algorithm}

In [16], it is proposed a rearrangement in a given Kronecker product matrix in order to achieve a rank-1 matrix. Given a matrix $\mathbf{A}=\mathbf{B} \otimes \mathbf{C} \in \mathbb{C}^{M_{1} M_{2} \times N_{1} N_{2}}$, where $\mathbf{B} \in \mathbb{C}^{M_{1} \times N_{1}}$ and $\mathbf{C} \in \mathbb{C}^{M_{2} \times N_{2}}$, we can see $\mathbf{A}$ as

$$
\mathbf{A}=\left[\begin{array}{cccc}
\mathbf{A}_{11} & \mathbf{A}_{12} & \ldots & \mathbf{A}_{1 N_{1}} \\
\mathbf{A}_{21} & \mathbf{A}_{22} & \ldots & \mathbf{A}_{2 N_{1}} \\
\vdots & \vdots & \ddots & \vdots \\
\mathbf{A}_{M_{1} 1} & \mathbf{A}_{M_{1} 2} & \ldots & \mathbf{A}_{M_{1} N_{1}}
\end{array}\right], \mathbf{A}_{i j} \in \mathbb{C}^{M_{2} \times N_{2}}
$$

The rearrangement $\mathcal{R}(\mathbf{A})$ of the matrix $\mathbf{A}$ is given by

$$
\mathcal{R}(\mathbf{A})=\left[\begin{array}{c}
\mathbf{A}_{1} \\
\mathbf{A}_{2} \\
\vdots \\
\mathbf{A}_{N_{1}}
\end{array}\right], \mathbf{A}_{j}=\left[\begin{array}{c}
\operatorname{vec}\left(\mathbf{A}_{1 j}\right)^{T} \\
\operatorname{vec}\left(\mathbf{A}_{2 j}\right)^{T} \\
\vdots \\
\operatorname{vec}\left(\mathbf{A}_{M_{1} j}\right)^{T}
\end{array}\right], j=1, \ldots, N_{1}
$$

We note that $\mathcal{R}(\mathbf{A}) \in \mathbb{C}^{M_{1} N_{1} \times M_{2} N_{2}}$.

In the present case, we can estimate $\mathbf{W}$, defined in the previous subsection, by a least-squares algorithm. Once we cannot assure that $\mathcal{R}(\mathbf{W})$ is a rank-1 matrix (due to the presence of the global channel in its product), we cannot estimate the matrices $\mathbf{S}$ and $\mathbf{H}^{\left(R_{2} D\right)}$ with a single SVD (optimal case), so we will estimate them with several SVDs using $\mathbf{W}_{m}$ defined as

$$
\mathbf{W}_{m} \leftarrow\left[\begin{array}{c}
\operatorname{vec}\left(\mathbf{W}_{1 m}\right)^{T} \\
\vdots \\
\operatorname{vec}\left(\mathbf{W}_{N m}\right)^{T}
\end{array}\right]
$$

where $\mathbf{W}_{n m}$ is the $n m^{t h}$ sub-block of $\mathbf{W} \in \mathbb{C}^{M_{D} N \times M_{R_{2}} M_{S}}$.

This procedure, denoted by Kronecker Rearrangement (KR), is based on the fact that $\mathbf{W}_{m}$ is a rank-1 matrix, which means that $\mathbf{S}_{. m}$ and $\mathbf{H}^{\left(R_{2} D\right)}$, with $m=1, \ldots, M_{S}$, are optimally estimated as the first left- and right-singular vectors of $\mathbf{W}_{. m}$, respectively.

At the end, there will be $M_{S}$ estimations of $\mathbf{H}^{\left(R_{2} D\right)}$. We take the first of these estimations. The estimated parameters have the ambiguities described in the previous subsection. The LS-KR algorithm is summarized in Algorithm 2.

\section{Identifiability Conditions}

Once the proposed algorithms are non-iterative, we do not need to worry about the uniqueness conditions. Regarding the identifiability issues, choosing a $\mathbf{G}_{1}$ matrix with equal rows, $\mathbf{G}_{2}$ and $\mathbf{G}_{0}$ matrices such that $\mathbf{G}_{02}$ has a right inverse, will assure that the algorithms will provide identifiable estimations. $P \geq M_{R_{2}} M_{S}$ is then a necessary condition.

\section{Simulations Results}

In this section, simulation results that evaluate the performance of the proposed receivers by means of Monte Carlo samples are presented. The metrics of performance used are both the Symbol Error Rate (SER) and the channels Normalized Minimum Square Error (NMSE). The number of Monte Carlo samples is equal to 15000 . The coding matrices $\mathbf{G}_{0}$, $\mathbf{G}_{1}$ and $\mathbf{G}_{2}$ are chosen to be i.i.d with zero mean complex

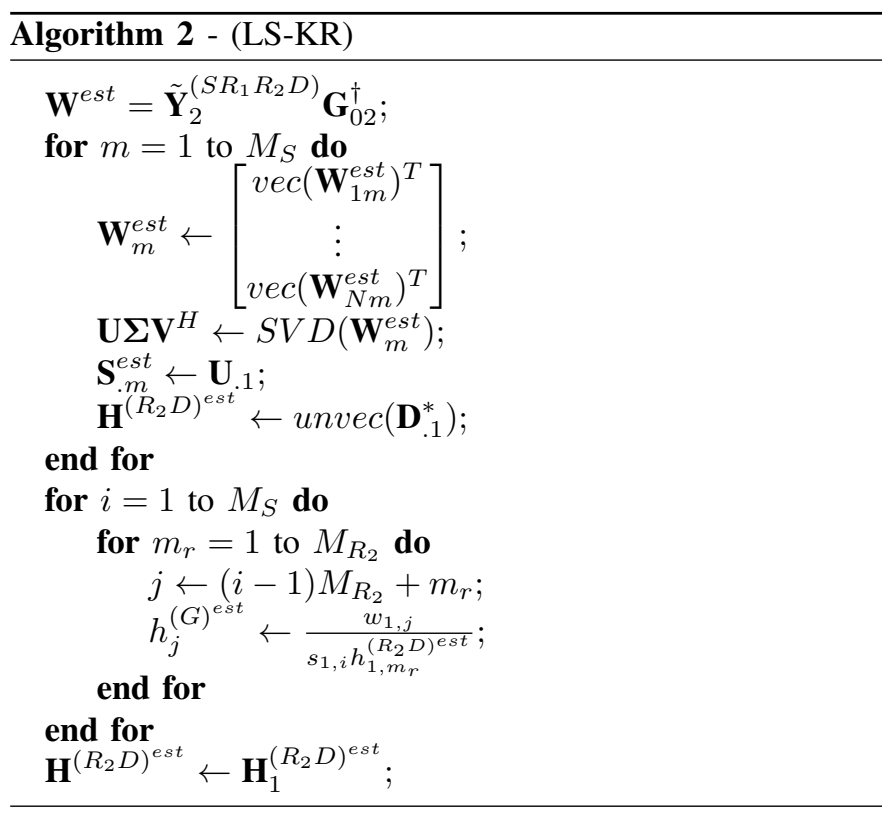

Gaussian distributions, however, $\mathbf{G}_{1}$ has equal rows and 16QAM is used. When stated otherwise, the number $P$ of transmission blocks is chosen to be 8 , while the number of symbols $N$ is equal to 100 . The number of antennas at the source, at the relays and at the destination are chosen to be 2 . The total transmission power is equal to 1 , and it is divided by the source and the relays. The path-loss coefficient $n$ is chosen to be 4 and it is considered Rayleigh fading Channels.

Figure 2 shows how the SER behaves as the SNR increases varying the number of antennas at the destination. We can see that the LS-KR receiver provides a better SER if comparing to the LS-KF receiver. This is due to the following reason. When we estimate $\mathbf{S}$ via SVD taking only the first column of the singular matrices, we are eliminating the noise subspace. The optimal case would be to estimate the parameters using a single SVD, once the LS-KF uses $M_{R_{2}} M_{S}$ SVDs and the LS-KR uses only $M_{S}$ SVDs, we can see that the LS-KR one uses less SVDs, being more close to the optimal case, providing, this way, a better performance. Also, we can see that as the number of antennas at the destination increases, the receivers provide a smaller SER, this is a benefit of the additional spatial diversity. Figure 3 shows how the NMSE of the channels behaves as the SNR increases. We can see that both receivers provide a better estimation of $\mathbf{H}^{\left(R_{2} D\right)}$ in comparison to the global channel $\mathbf{H}^{(G)}$. This occurs because we cannot estimate the global channel via SVD, we can only estimate it using the noisy matrix W. Also, we can see that the LS-KR algorithm provides a better performance regarding $\mathbf{H}^{\left(R_{2} D\right)}$ due to the reason explained before. The performance regarding the global channel is practically the same, once it is estimated for both algorithms using the same equation.

Comparing with the two-hop scenario, i.e, the scenario which a single relay is used, Figure 4 shows how the SER behaves as the SNR increases. In this figure, the receivers used in the two-hop case are based on (4) and (5), being very similar to the LS-KF and LS-KR algorithms. However, due to lack of space we didn't show the expressions of these 


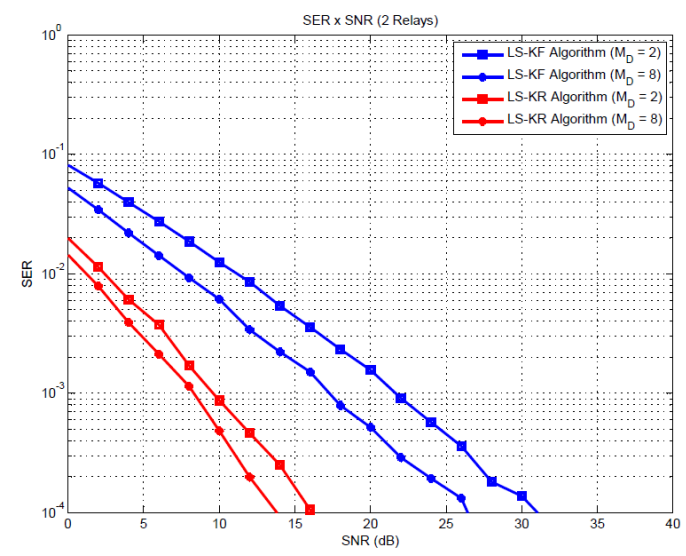

Fig. 2: SER versus SNR.

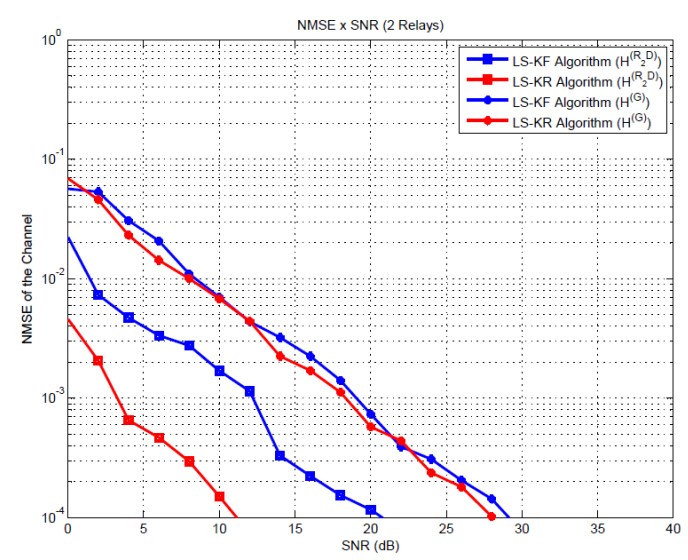

Fig. 3: NMSE versus SNR.

algorithms. We can see in Figure 4 that the three-hop scenario provides smaller SERs. This is expected, because, once we use more relays, we will have shorter distances between the nodes, needing less transmission power to communicate.

\section{CONCLUSION}

The major contribution of this work is the proposal of two semi-blind receivers for one-way three-hop cooperative MIMO AF relay systems. It was shown that this transmission scheme satisfies a PARATUCK-3 tensor model. The proposed receivers use the KRST coding at the source and an AF coding at the relays. One of these receivers is based on a factorization of the Kronecker product, while the other is based on a rearrangement of this Kronecker product. Simulation results have shown that the three-hop scenario provides a better performance, regarding the SER, compared to the twohop scenario, due to the path-loss gain. Simulation results also showed that the LS-KR algorithm provides a better performance than the LS-KF one.

In future works, we aim to analyze the complexity of the proposed algorithms and generalize them for the case of $K$ relays. The development of a receiver based on the Alternating Least Squares (ALS) algorithm is also a perspective.

\section{REFERENCES}

[1] K.J.R. Liu, A.K. Sadek, W. Su, and A. Kwasinski, Cooperative Communications and Networking. Cambridge University Press, 2009.

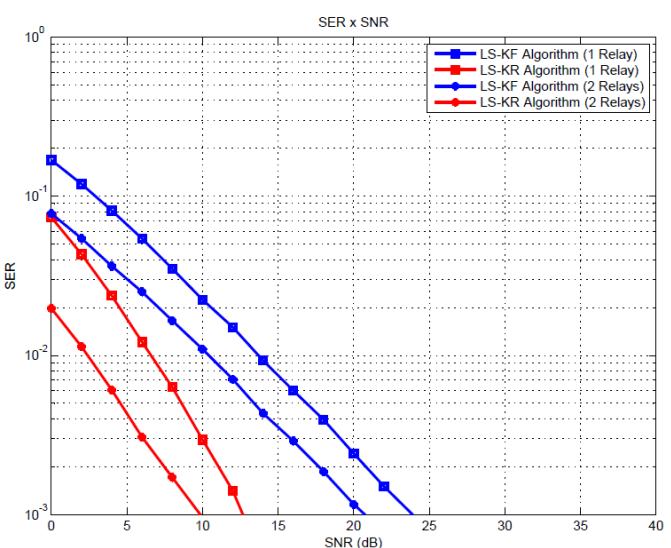

Fig. 4: Comparison of the SER versus SNR for 1 and 2 relays.

[2] S. Plevel, S. Tomazic, T. Javornik, and G. Kandus, "MIMO: Wireless Communications", Encyclopedia of Wireless and Mobile Communications, DOI: 10.1081/E-EWMC-120043484. 2008.

[3] N. D. Sidiropoulos, R. Bro e G. B. Giannakis, "Parallel Factor Analysis in Sensor Array Processing", IEEE Transactions on Signal Processing, vol. 48, no. 8, pp. 2377-2388, Aug. 2000.

[4] N. D. Sidiropoulos, G. B. Giannakis e R. Bro, "Blind PARAFAC Receivers for DS-CDMA Systems", IEEE Transactions on Signal Processing, vol. 48, no. 3, pp. 810-823, Mar. 2000.

[5] A. de Almeida, G. Favier, "Double Khatri-Rao Space-Time-Frequency Coding Using Semi-Blind PARAFAC Based Receiver", IEEE Signal Processing Letters, May. 2013.

[6] A. L. F. de Almeida, G. Favier e J. C. M. Mota, "PARAFAC-Based Unified Tensor Modeling of Wireless Communication Systems with Application to Blind Multiuser Equalization", Signal Processing - Special Issue on Tensor Signal Processing, vol. 87, no. 2, pp. 337-351, Feb. 2007.

[7] C. A. R. Fernandes, A. L. F. de Almeida e D. B. da Costa, "United Tensor Modeling for Blind Receivers in Multiuser Uplink Cooperative Systems", IEEE Signal Processing Letters, vol. 19, no. 5, pp. 247-250, 2012.

[8] A. L. F. de Almeida, C. A. R. Fernandes e D. B. da Costa, "Multiuser Detection for Uplink DS-CDMA Amplify-and-Forward Relaying Systems", IEEE Signal Processing Letters, vol. 20, no. 7, pp. 697-700, Jul. 2013.

[9] L. R. Ximenes, A. L. F. de Almeida, G. Favier, "PARAFAC-PARATUCK Semi-Blind Receivers for Two-Hop Cooperative MIMO Relay Systems", IEEE Transactions on Signal Processing, vol. 62, no. 14, July 15, 2014

[10] L. Ximenes, G. Favier, A. de Almeida, "Semi-Blind Receivers for NonRegenerative Cooperative MIMO Communications Based on Nested PARAFAC Modeling”, IEEE Transactions on Signal Processing, Sep. 2015.

[11] G. Favier, C. A. R. Fernandes e A. L.F. de Almeida, "Nested Tucker Tensor Decomposition with Application to MIMO Relay Systems Using Tensor Space-time Coding (TSTC)", Signal Processing, no. 128, pp. 318-331, Nov. 2016.

[12] M. N. da Costa, G. Favier, A. L. F. de Almeida e J. M. T. Romano, "Tensor Space-Time (TST) Coding for MIMO Wireless Communication Systems", Signal Processing, vol. 92, no. 4, pp. 1079-1092, 2012.

[13] A. de Almeida, G. Favier, L. Ximenes, "Space-time-frequency (STF) MIMO communication systems with blind receiver based on a generalized PARATUCK2 model", IEEE Transactions on Signal Processing, Apr. 2013.

[14] N. D. Sidiropoulos and R. S. Budampati, "Khatri-Rao space-time codes", IEEE Transactions on Signal Processing, vol. 50, no. 10, pp. 2396-2407, Oct. 2002.

[15] R. A. Harshman, M. E. Lundy, "Uniqueness Proof for a Family of Models Sharing Features of Tucker's Three-Mode Factor Analysis and PARAFAC/CANDECOMP”, Psychometrika, vol. 61, no. 1, pp. 133-154, Mar. 1996.

[16] C. Van Loan and N. Pitsianis, "Approximation with Kronecker Products", Linear Algebra for Large Scale and Real-Time Applications, pp. 293-314. Cornell University. 1993. 